\title{
Using Multiple Criteria Decision Analysis to Aid the Selection of Enterprise Resource Planning Software: A Case Study
}

\author{
Ross Parkhill ${ }^{1}$, Valerie Belton ${ }^{1}$, Umit Bititci ${ }^{1}$, Alan Roberts ${ }^{2}$, and Marisa Smith ${ }^{1}$ \\ ${ }^{1}$ University of Strathclyde, Glasgow, UK \\ e-mail: ross.parkhillastrath.ac.uk \\ ${ }^{2}$ BHC Ltd., Medwyn Park, Carnwath, ML11 8HS
}

\begin{abstract}
BHC Ltd is a family owned SME which specialises in steel fabrication for the construction industry. Due to rapid growth over the past decade the company's current business software has evolved from a collection of semi-integrated individual packages and Excel spreadsheets. To help the company become more efficient during the current financial downturn and to ensure they are capable of future growth, BHC Ltd initiated a project with the University of Strathclyde to select and implement an Enterprise Resource Planning (ERP) solution.

This paper will provide a case study of BHC's ERP selection process. In particular it will discuss how steel specific business requirements and organisational culture led us to use multiple criteria decision analysis (MCDA) when making a final software selection. The MCDA process that was followed is further discussed and includes the success that was achieved by using this approach.
\end{abstract}

\section{Introduction}

This paper discusses activities undertaken as part of a Knowledge Transfer Partnership (KTP) No.6788 between BHC Ltd, a SME steel fabricator from South Lanarkshire and the University of Strathclyde, Glasgow.

The focus of this KTP project was on the design and implementation of an integrated software solution, in order to support estimating, planning and materials management. This was to be achieved through an ERP system, which will give BHC Ltd greater control and visibility of their processes allowing them to deliver jobs to site faster and more reliably, while also helping to facilitate the collection and analysis of data. This paper provides a case study of the methodology followed to select the correct ERP solution for BHC ltd, including the challenges that arose due to the culture within the organisation. The paper also discusses the use of multiple criteria decision analysis (MCDA) to aid the selection of an ERP solution and how this approach faired within the culture.

R.J. Howlett (Ed.): Innovation through Knowledge Transfer, SIST 5, pp. 39-49. springerlink.com

(C) Springer-Verlag Berlin Heidelberg 2010 


\section{Background}

\section{The Company}

BHC Ltd design, manufacture and build structural steel and steel frame buildings for the construction industry. Founded in 1992 by Brian and Marjorie Hewitt, the company has grown rapidly over the last decade, increasing both their manufacturing facilities and capabilities at their base in Carnwath, Scotland. Their current markets include retail, leisure, industrial and agricultural buildings. Projects range from standard single span warehouses which are designed, manufactured and installed by the company, to large construction projects where the business only supplies and erects the structural steel work.

BHC Ltd aim to consolidate the company during the current market downturn, but envisage the potential for a future 30\% growth in turnover. Beyond this the company plans growth into global markets (Europe and Asia) with additional manufacturing sites being added. To help ensure this growth is possible BHC have identified that they need to improve their current business software systems. The company has an existing MRP system and separate accounts package but this does not meet the business's growing needs for both volume and functional capabilities. The company's systems have not grown as the scope and volume of orders increased and a large amount of management time is spent on scheduling, manufacturing issues and manipulating current software or spreadsheets to retrieve vital data. The company made a decision in early 2009 that they would look to ERP software to eradicate these issues, initially considering building their own software before deciding that an 'off-the-shelf' solution was more viable.

\section{Company Culture}

It is widely recognised that different organisations can have distinctive cultures; the type of culture present within a company plays an important role in the ERP selection and implementation processes. Organisational culture itself is commonly described as 'the way we see and do things around here', and while this may be a simple statement it gives a good indication of how organisational culture can affect this type of project.

BHC is a family owned SME that can be categorised as an owner-manager organisation, where Brian Hewitt operates as both Managing Director and main stakeholder. Research into this type of organisation shows a firms' culture may be heavily influenced by the motivations, attitudes and management style of its owner-manager (Butler, 2006). Brian Hewitt is entrepreneurial in his approach to business. His own personal drive has not only been the main catalyst for company growth but also the reason BHC have successfully branched off into other nonsteel opportunities. Brian Hewitt has achieved this success with a 'get up and go' attitude to business management often without any form of strategic planning.

The culture within BHC Ltd could be categorised as a 'power culture', based on the dominance of one or a small number of individuals within an organisation. They make the key decisions for the organisation (Handy, 1985). BHC Ltd does not have a board of directors or senior management steering groups to discuss and 
agree on business decisions or future strategy, all major decisions are taken by Brian Hewitt.

\section{ERP Software}

ERP systems are enterprise-wide software systems that support seamless integration of processes across functional areas including accounting, manufacturing planning and scheduling, supply chain management, human resources, Customer Relationship Management (CRM) and project management. ERP systems are designed to provide improved workflow, standardisation of business practices, and access to real-time information through the use of a common database for data storage.

Selecting an inappropriate ERP system is a major reason for ERP implementation failure. Due to the complexity of the business environment and the diversity of ERP alternatives, system selection can be a monotonous and lengthy task. Given the considerable financial investment and potential risks and benefits, the importance of selecting a suitable ERP system cannot be overemphasised since it is a decision on how to shape the organisational business (Teltumbde, 2000). Once the correct ERP system has been identified the process of actually implementing the software can begin. ERP systems are complex and implementing one can be a challenging, time-consuming and expensive project for any company (Davenport, 1998). Several things can affect the success of an implementation, these critical success factors (CSF's) include: top management support, vendor support, consultant competence, user support, IT capability and project manager leadership (Wang et al, 2008).

The selection and implementation of a suitable ERP system for BHC was given a two year project plan based on similar ERP projects that the University had managed. Although both these stages can provide their own difficulties, the selection of the correct software is critical to the overall success of the project. Often it can be extremely difficult to differentiate between ERP solutions due to the complexity of numerous considerations that should be taken into account. Therefore it was decided we would perform a multiple criteria decision analysis (MCDA) to help ensure BHC followed a structured decision-making approach.

\section{Multiple Criteria Decision Analysis (MCDA)}

MCDA can be defined as a collection of formal approaches which seek to take explicit account of multiple criteria in helping individuals or groups explore decisions that matter (Belton \& Stewart, 2002). One of the principle aims of MCDA approaches is to help decision makers organise and synthesise information in a way that leads them to feel comfortable and confident about making a decision, minimising the potential for post-decision regret by being satisfied that all criteria or factors have been properly taken into account (Belton \& Wright, 2005).

Various models exist that can be used in a MCDA problem; these include value measurement models, reference level models and outranking models. Regardless of which style of model is selected a MCDA methodology can be used to: 
- Take explicit account of multiple, conflicting criteria in aiding decisionmaking

- Help structure a problem, providing focus and a language for discussion

- Allow decision makers to learn about the problem situation, about their own and others values and judgments

- Provide an audit trial for a decision

The three key phases of the MCDA process are problem identification and structuring, model building and use, and the development of action plans. The selection process for determining the most appropriate ERP software among a set of possible alternatives on the market is a multi-criteria decision making (MCDM) problem (Karsak \& Ozogul, 2009). Within BHC a MCDA methodology was used to aid the final decision making process.

\section{A Case Study of Selecting an ERP Solution for BHC Ltd.}

\subsection{Process Analysis and Redesign}

This initial project stage was aimed at redesigning the business processes within BHC. As the company has grown, many of their current processes and procedures have developed without considering optimisation or best practice approaches. The current system is greatly hindered by the number of disjointed software systems the company utilises.

\section{Value Stream Mapping}

Value stream mapping is a Lean technique used to analyse and map all the physical and information flows required to bring a product or service to a consumer. The tool was selected for BHC as it provides a clear way to illustrate the current and future state of a process. It is called a value stream map because it depicts the value-adding activities necessary to produce what the customer requires, in the sequence in which they happen.

In BHC we followed the standard process of firstly creating a current state map of the organisation. To achieve this we divided the full business into manageable subsections; Order Entry/Estimate/Tender, Purchase, Manufacture, Erect, Accounts. Taking each area individually we asked key personnel from associated departments along with a core project team, which was made up of key BHC individuals who had a broad overall business knowledge, to explain how the current process worked. This was drawn on a large piece of paper using 'post-it' notes to allow for quick and easy changes or additions. The current map detailed how each subsection functioned including all the interactions with other departments and how and where they use any software.

Once the current state was mapped, we brought the same groups back together to analyse the processes taking place. The aim of this was to identify inefficiencies like double handling and evaluate the use of the current software. From this we 
created a future state map, indicating how each subsection could operate more efficiently supported by improved software functionality.

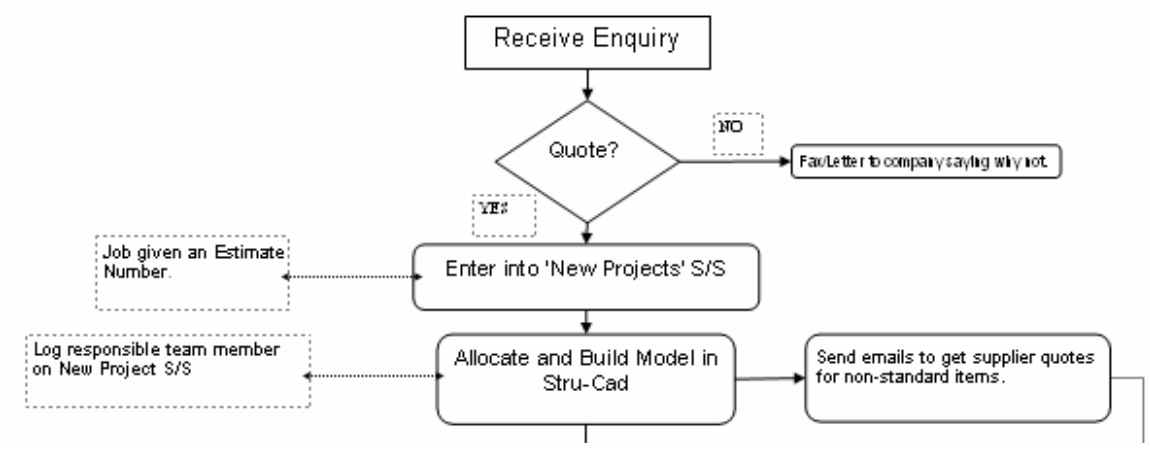

The task of business process design, if carried out correctly, builds consensus about how the business will work in the future and makes clear the mechanisms through which pay-back will be delivered. This makes it much easier to identify essential software functionality and separate it from "nice to have" features. It also enrolls staff in the project (Okrent \& Vokurka, 2004).

\subsection{Requirements Specification}

Redesigning the processes before selecting a software solution provided a solid foundation for developing a statement of requirements. Within BHC we gathered information throughout both the current and future mapping workshops, detailing areas that were highlighted as important requirements for BHC. The project team then met and produced the 'BHC ERP Business Process Requirements' document that summarised the major needs from each subsection of the business. It was agreed that this would form the basis of the full specification that would be sent out as part of the 'Invitation to Tender'. The full specification would be produced concurrently as we carried out the initial market research and vendor search.

\subsection{Initial Market Search and Suppliers Selection}

As BHC had initially considered writing their own ERP solution they had no prerequisites as to what ERP software they wanted to implement. Therefore the market research involved looking at the full spectrum of solutions on offer; this included full ERP solutions, modular software providers (accounts package, project management, planning and scheduling software) and steel-specific $\mathrm{MRP} /$ document management software. The internet provided much of the initial leads with several websites like the Evaluation Centre and the Technology Evaluation Centre providing excellent comparisons and software reviews. Individual supplier websites were also reviewed for case studies of companies similar to BHC or 
for experience in the construction or metals sector. Advice was also received from other KTP associates throughout the UK involved in ERP projects and from experienced University of Strathclyde staff. Research of what software other steel construction firms used was also carried out. Contact was made with all the larger members of the British Constructional Steel Association and telephone interviews held with IT or Production leaders.

From this initial research nine ERP software providers were identified, Epicor, Visibility, SAP, EFACS, IFS, M1, Infor, Microsoft Dynamics, Oracle and one steel-specific MRP provider StruMis. Each of these companies were provided with the 'BHC ERP Business Process Requirements' and asked to demonstrate their software on-site at BHC or provide a web demo. While this process was time-consuming with each demonstration taking up most of a day it allowed the project team to get an excellent feel for each piece of software as well as meet representatives of each company.

\subsection{The Invitation to Tender (ITT)}

During the software search and demonstration phase we continued to augment the BHC ERP Business Process Requirements document as we became more knowledgeable not only about our own true requirements but also by what we wanted from ERP software. To turn this into a full specification, input was received from key members of each department as well as senior management. The specification was then combined with details of the project and BHC data samples to form an ITT. This was sent to the ten software providers and each was asked to respond within a 3 week time scale.

We received nine responses to the ITT, and one further provider removed themselves from the running after further investigation into the solution required. Of the eight remaining, each response was evaluated individually against their ability to meet the key functional needs of BHC. All the information was analysed by the project team who then presented a shortlist to senior management that included three ERP providers and the steel specific solution. Both the initial demonstrations and the responses to the ITT identified that due to the nature of BHC's business, providing a fully functional solution was going to be complex process requiring several substantial changes to the 'out of the box' solution usually offered. At this point it was agreed by the project team and senior management that we would be unable to make a final selection from the evidence we had seen so far and that a second round of more in-depth demonstrations should take place.

\subsection{Software Workshops}

To aid the final selection process we needed to delve deeper into the functionality each software could offer and in particular understand how each solution would deal with the core requirements of BHC's business. These specific steel industry processes were not something any of the full ERP systems could deal with as standard and due to this there was a feeling of risk towards going with this type of 
solution. However the steel specific MRP solution, while handling core requirements well, lacked additional functionality which would result in a final solution with multiple software packages connected together.

To help elevate fears of using a full ERP system we sent each of the three ERP providers a full package of data that included a complete BHC project and asked them to demonstrate how this job would move through their software. This was an extensive test and each workshop was held over two days within the BHC plant. We also decided to use this opportunity to involve more BHC staff as we realised this would help with the change management process during and after implementation. Working with each department and utilising the specification we identified what the critical functionality was for each department. We then produced a scoring sheet and asked each department to send representatives to the appropriate section of the workshop and score each piece of software. This allowed future end users to directly contribute towards the final selection and helped promote a feeling of ownership towards the decision, this is something that could prove vital during the difficult stage of implementation and go live.

\subsection{Final Software Selection Process}

Although the final workshops were beneficial for various reasons we still could not differentiate significantly enough between each software package to select a final solution. Each individual software solution had its own particular merits or shortcomings, however it was proving difficult to view all these considerations as a whole and compare each overall package against each other. Also, as the process to this point had been extremely time-consuming senior management had been unable to attend all the reference site visits and workshops. Although the project team were confident that an ERP solution could be achieved, the steel specific solution was being increasingly considered as a reduced risk option.

At this point we decided to utilise our links with the University and carryout a multiple criteria decision analysis (MCDA). There was some concern about attempting MCDA within BHC as the company had no experience in this approach and it was not normal to BHC's culture. However as this was a KTP project the University wished to ensure that BHC were exposed to well-founded decisionmaking processes and followed a structured methodology to arrive at an evidencebased justifiable selection. However, it is important to recognise that MCDA is an aid to decision making and that the 'preferred' outcome depends on the values of the decision makers. It would therefore be down to the management to decide whether they wished to go with the software identified through the MCDA, or to review the priorities reflected in the model.

To aid the MCDA process we used Web $\mathrm{V} \bullet \mathrm{I} \bullet \mathrm{S} \cdot \mathrm{A}$ software, a multi-criteria decision support system which is based on a multi-attribute value function (www.simul8.com). The use of such an approach greatly facilitates the consideration of differing values and opinions through the provision of a focus for exploration and discussion of a problem (Belton, Ackermann \& Shepherd, 1997). Through a serious of meetings with BHC management and University staff we used a top-down approach to identify the following six high level criteria: 
- Cost - including software licenses, implementation costs, maintenance and support costs

- Software functionality - as demonstrated in workshops

- Confidence in meeting critical/core functionality

- Software flexibility and configurability

- Reseller considerations - including risk, staff personality, support

- Implementation strategy

These were then broken down further into sub-criteria which we could score each of our four final solutions against. This information was collated utilising the Web $\mathrm{V} \cdot \mathrm{I} \cdot \mathrm{S} \bullet \mathrm{A}$ software to form a decision value tree.

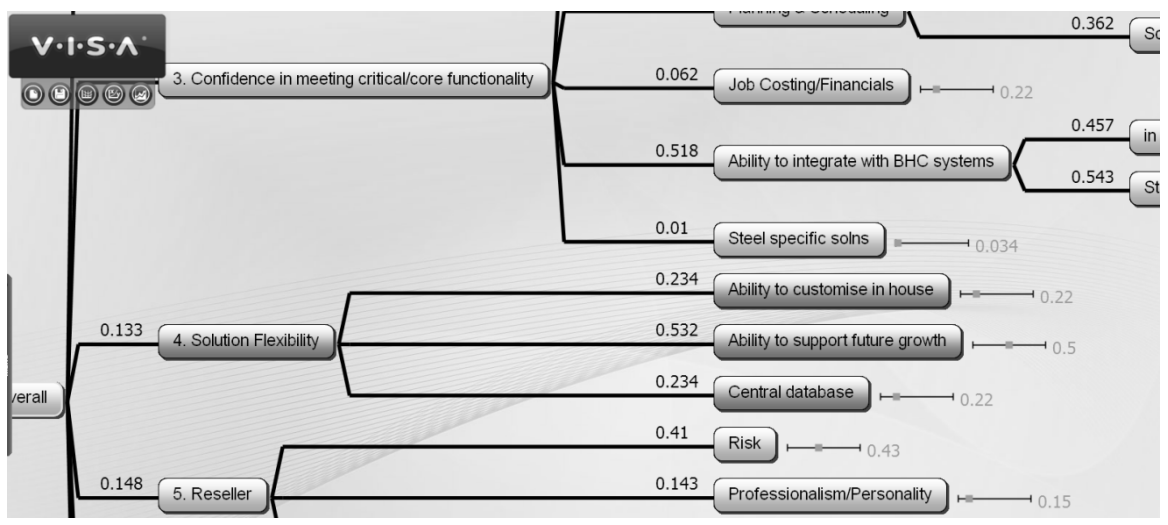

We then brought all the major project stakeholders together for a half day decision-making session. This was held in the University to provide focus and minimise disturbances. Using the $\mathrm{Web} \mathrm{V} \bullet \mathrm{I} \bullet \mathrm{S} \bullet \mathrm{A}$ value tree, as a group we went through each sub-criterion scoring the four possible solutions. A zero to one hundred scoring system had been agreed at the start of the meeting with clarification on how the system should operate. The process of scoring each sub-criterion generated enthusiastic group discussion, with many questions and system challenges being raised.

Each of the sub-criteria and six high level criteria were then weighted to reflect their 'importance' against BHC's ERP requirements and overall business needs (where the notion of 'importance' captures the relative value of moving from 0 to 100 on each criterion). This stage provided further interesting debate as many of the stakeholders had differing views as to what they deemed the most important factors. This process provided a platform for everyone to express their views and helped provide clarity on what was important to $\mathrm{BHC}$ as a whole. In particular it allowed BHC management to address what had now become the two key issues:

- The risk involved in selecting an ERP solution as apposed to the steel specific software

- Quantify the benefits additional ERP functionality can bring to BHC 
Once the scoring and weighting process was complete $\mathrm{Web} \mathrm{V} \cdot \mathrm{I} \bullet \mathrm{S} \bullet \mathrm{A}$ software was used to show the results graphically. This visual representation showed clearly the ranking of how each solution performed. Further experimentation with different scenarios was carried out, in these 'what if' scenarios we changed the weighting of key criteria and reviewed how this affected the overall results. This process enabled us to prove the original findings as even when changing various weightings one supplier continued to perform strongly.

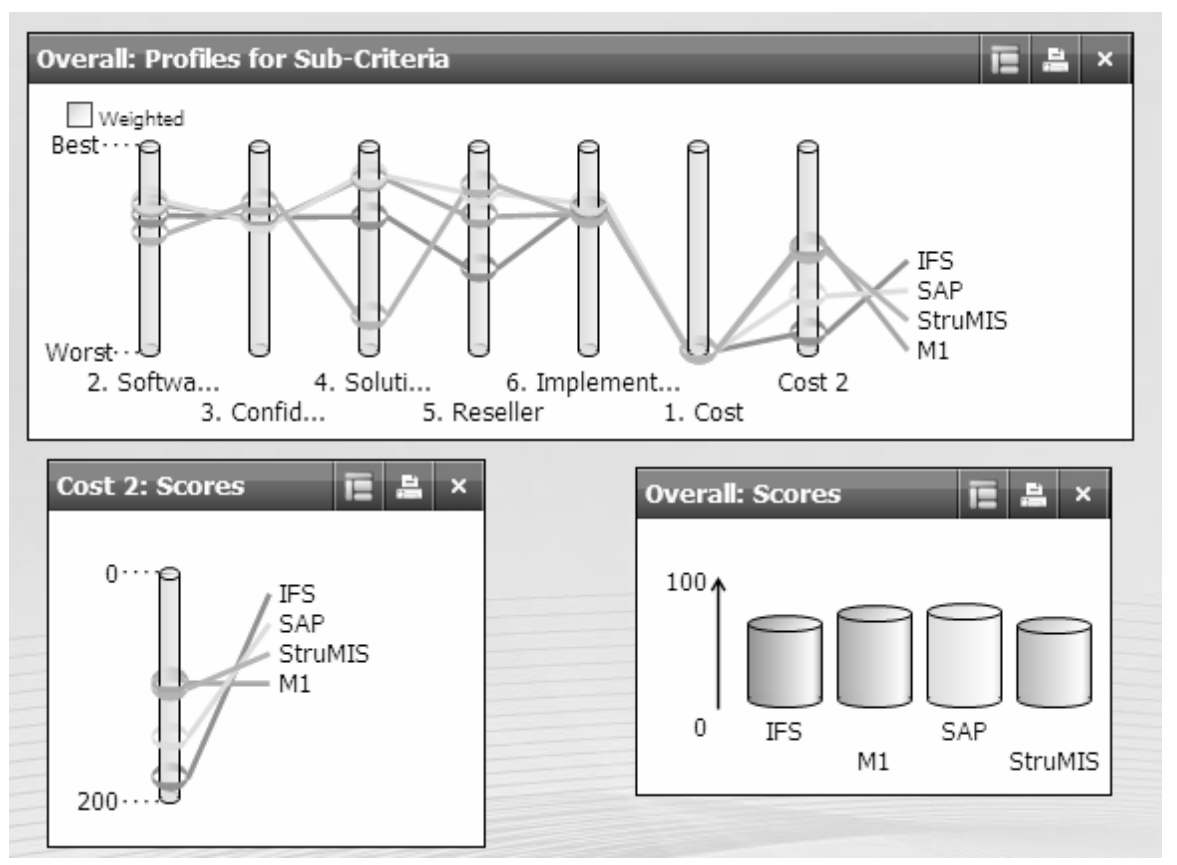

At the close of the meeting it was agreed by all stakeholders that we had now identified a preferred supplier for the management to consider.

\section{Discussion and Conclusion}

This case study detailed the steps undertaken to select an ERP solution for BHC Ltd. Although we followed a widely accepted methodology, complexities arose due to the nature of the business and culture that exists within the organisation. Full ERP systems are not common within the steel industry; throughout this project we could not find another UK or European steel fabricator with an 'off the shelf' solution in place. Due to this, many of the steel specific problems had never been tackled by any of the ERP providers we approached. This made evaluating each piece of software extremely difficult and led to an extended period of market research and workshop testing. 
The inability of potential suppliers to demonstrate fully a system that could meet BHC's requirements made senior management nervous and resulted in the strengthening of the case to select the steel specific Material Requirements Planning (MRP) software. However this solution would only provide some of the benefits that BHC initiated this project to deliver. Investigating ERP software and attending reference site visits or software workshops proved to be a very timeconsuming task, therefore Brian Hewitt was unable to make all the scheduled appointments. This resulted in the project team being empowered to drive the project and find a suitable solution. However as the final decision date drew closer there was uncertainty around the correct way forward as even the project team could not unanimously decide on a preferred solution. This was mainly due to the multiple, often conflicting, criteria that must be considered when selecting an ERP system.

To help with the decision process and ensure BHC followed a structured approach when identifying the correct solution we turned to Multiple Criteria Decision Analysis (MCDA). There were concerns about using such an approach due to the organisational culture and normal decision-making process within BHC Ltd. However this process proved to be a massive success with excellent feedback from BHC management and members of the project team. With the aid of Web $\mathrm{V} \cdot \mathrm{I} \bullet \mathrm{S} \bullet \mathrm{A}$ software we were able to structure a complex decision and provide a platform for extensive discussion on the correct software choice. This lead to a better considered, justifiable and explainable decision being made; something that could prove invaluable during the difficult stages of implementation and the change management process.

This type of formal decision-making is not normal to BHC's culture; however the process of MCDA proved to have added value in this type of organisation as it forced key BHC individuals to think and discuss a major decision through as a team. The MCDA process ensured that company owners could now make a final selection with confidence and a full understanding of why the ERP software was the correct decision for the business.

\section{References}

Belton, V., Ackermann, F., Shepherd, I.: COPE-ing with V·I·S·A: Integrated Support from Problem Structuring through to Alternative Evaluation. Journal of Multicriteria Decision Analysis (1997)

Belton, V., Stewart, T.J.: Multiple Criteria Decision Analysis: An Integrated approach. Kluwer Academic Publishers, Dordrecht (2002)

Belton, V., Wright, G.: Making Decisions, The Graduate School of Business, University of Strathclyde (2005)

Butler, D.: Enterprise Planning and Development: small business start-up survival and growth. Butterworth-Heinemann, Butterworths (2006)

Davenport, T.H.: Putting the enterprise into the enterprise system. Harvard Business Review (1998)

Handy, C.B.: Understanding Organizations, 3rd edn. Penguin Books, Harmondsworth (1985) 
Karsak, E., Ozogul, C.: An integrated decision making approach for ERP system selection. Expert Systems with Applications 36 (2009)

Okrent, M., Vokurka, R.J.: Process mapping in successful ERP implementations. Industrial Management + Data Systems (2004)

Teltumbde, A.: A framework for evaluating ERP projects. International Journal of Production Research (2000)

Wang, E., Shih, S., Jiang, J., Klein, G.: The consistency among facilitating factors and ERP implementation success: A holistic view of fit. The Journal of Systems and Software 81 (2008) 
